\title{
To use or not to use new IT: the effect of healthcare professional's OCB on intention to use new clinical IT
}

\begin{abstract}
Although compared to other industries the utilization of information technology (IT) has slowly increased in many healthcare organizations, a variety of IT systems in the forms of clinical information systems, personal digital assistants, electronic patient records and other applications have gradually become established in the healthcare industry. IT has been enhanced as a means to advance healthcare services and patient care. Yet, it is impractical to imagine that if a new clinical information system is adopted by a healthcare organization all organizational members will be willing to apply the system simply. As healthcare professionals play an important role in the healthcare industry, there is no doubt that introducing new medical IT should be coupled with healthcare professionals' acceptance to use the IT properly. Clinical IT applications in healthcare are regarded as a key element in raising the quality of medical care. However, factors affecting the healthcare professionals' adoption behavior regarding the clinical IT systems are still not completely clear. In this study, by integrating the professional characteristics of healthcare profession with technology acceptance model, a modified model has been developed to address the issues of accepting new technology in the health care context. In this research (as a conceptual research) the concept of change-oriented Organizational Citizenship Behavior (OCB) is proposed as the key factor in the proposed conceptual framework concerning healthcare professionals' IT adoption behavior.
\end{abstract}

Keyword: Change-oriented OCB; Clinical IT systems; Computer self-efficacy; Perceived threat to professional autonomy; TAM 